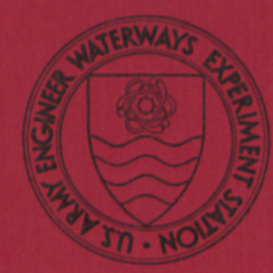

\title{
ENDOCHRONIC CONSTITUTIVE LAW FOR LIQUEFACTION OF SAND
}

\author{
by \\ Zdenek P. Bazant and Raymond J. Krizek \\ The Technological Institute, Department of Civil Engineering \\ Northwestern University, Evanston, III. 60201
}

August 1976

Final Report

Approved For Public Release; Distribution Unlimited

ב

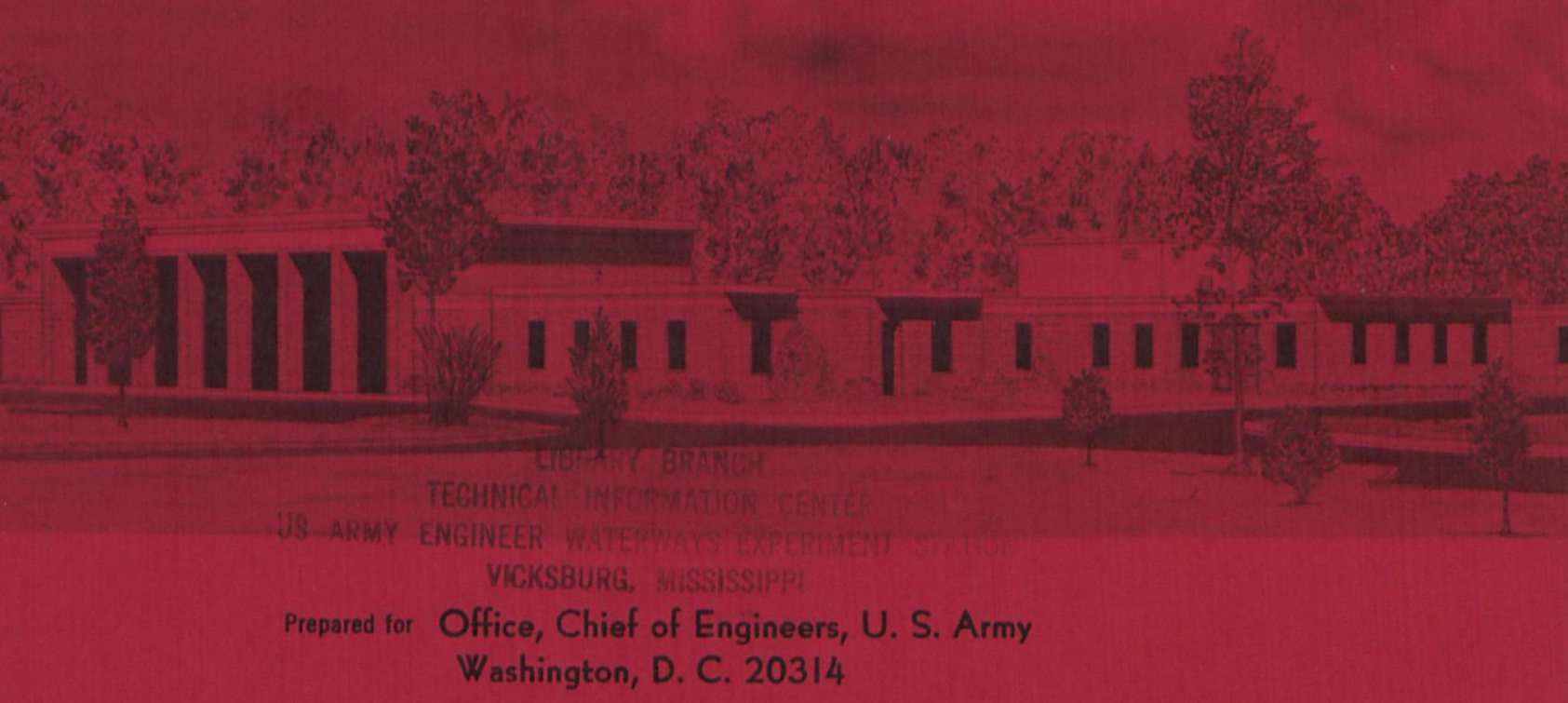

Under Contract No. DACW39-75-M-3739

Monitored by Soils and Pavements Laboratory

U. S. Army Engineer Waterways Experiment Station

P. O. Box 631, Vicksburg, Miss. 39180 


\begin{tabular}{|c|c|}
\hline REPORT DOCUMENTATION PAGE & $\begin{array}{c}\text { READ INSTRUCTIONS } \\
\text { BEFORE COMPLETING FORM }\end{array}$ \\
\hline $\begin{array}{l}\text { 1. REPORT NUMBER } \\
\text { Contract Report S }-76-9\end{array}$ & 3. RECIPIENT'S CATALOG NUMBER \\
\hline \multirow{2}{*}{$\begin{array}{l}\text { 4. TITLE (and Subill!) } \\
\text { ENDOCHRONIC CONSTITUTIVE LAW FOR LIQUEFACTION } \\
\text { OF SAND }\end{array}$} & $\begin{array}{l}\text { 5. TYPE OF REPORT \& PERIOD COVERED } \\
\text { Final report }\end{array}$ \\
\hline & 6. PERFORMING ORG. REPORT NUMBER \\
\hline $\begin{array}{l}\text { 7. AUTHOR(*) } \\
\text { Zdenek P. Bazant } \\
\text { Raymond J. Krizek }\end{array}$ & 8. CONTRACT OR GRANT NUMBER(s) \\
\hline $\begin{array}{l}\text { 9. PERFORMING ORGANIZATION NAME AND ADDRESS } \\
\text { The Technological Institute } \\
\text { Department of Civil Engineering } \\
\text { Northwestern University, Evanston, IIl. } 60201\end{array}$ & $\begin{array}{l}\text { 10. PROGRAM ELEMENT.PROJECT. TASK } \\
\text { AREA \& WORK UNIT NUMBERS }\end{array}$ \\
\hline \multirow{2}{*}{$\begin{array}{l}\text { 11. CONTROLLING OFFICE NAME AND ADDRESS } \\
\text { Office, Chief of Engineers, U. S. Army } \\
\text { Washington, D. C. } 20314\end{array}$} & $\begin{array}{l}\text { 12. REPORT DATE } \\
\text { August } 1976\end{array}$ \\
\hline & $\begin{array}{l}\text { 13. NUMBER OF PAGES } \\
24\end{array}$ \\
\hline \multirow{2}{*}{$\begin{array}{l}\text { 14. MONITORING AGENCY NAME ADDRESS(It dillerent Irom Controlling Office) } \\
\text { U. S. Army Engineer Waterways Experiment Station } \\
\text { Soils and Pavements Laboratory } \\
\text { P. 0. Box 631, Vicksburg, Miss. } 39180\end{array}$} & $\begin{array}{l}\text { 15. SECURITY CLASS. (ot this roport) } \\
\text { Unclassified }\end{array}$ \\
\hline & $\begin{array}{l}\text { 15a. DECLASSIFICATION/DOWNGRADING } \\
\text { SCHEDULE }\end{array}$ \\
\hline \multicolumn{2}{|l|}{$\begin{array}{l}\text { 16. DISTRIBUTION STATEMENT (ol thl-Ropors)- } \\
\text { Approved for public release; distribution unlimited. }\end{array}$} \\
\hline \multicolumn{2}{|l|}{ 17. DISTRIBUTION STATEMENT (OI the abstract entered In Block 20, il dillerent from Report) } \\
\hline \multicolumn{2}{|l|}{ 18. SUPPLEMENTARY NOTES } \\
\hline $\begin{array}{ll}\text { Constitutive relations } & \text { Hysteresis } \\
\text { Cyclic loads } & \text { Liquefaction (soils) } \\
\text { Densification (soils) } & \text { Sands }\end{array}$ & \\
\hline \multicolumn{2}{|c|}{$\begin{array}{l}\text { 20. ABSTRACT (Coninue on roverso side "lnecossary and identify by block number) } \\
\text { A nonlinear constitutive law is developed to describe the densification and } \\
\text { hysteresis of sand under cyclic loading. This law, together with the pre- } \\
\text { viously established model for an inelastic two-phase medium, gives a realistic } \\
\text { prediction of the pore pressure buildup and associated liquefaction of sand } \\
\text { due to cyclic shear. The law is of the endochronic type and consists of quasi- } \\
\text { linear first-order differential equations expressed in terms of intrinsic time, } \\
\text { which is an independent variable whose increments depend on the strain incre- } \\
\text { ments. This accounts for the accumulation of particle (Continued) }\end{array}$} \\
\hline EDITION OF I NOV 65 IS OBSOLETE & Unclassified \\
\hline
\end{tabular}


20. ABSTRACT (Continued). rearrangements, which are characterized by a parameter termed the rearrangement measure. The basic extensions of this work with regard to the application of endochronic theory to metals are due to the fact that sands densify upon shearing and are sensitive to confining stress; these two features are shared with the formulation of endochronic theory for concrete. Several typical examples are given to illustrate the ability of the developed theory to adequately characterize the densification, hysteresis, and liquefaction tendency of sands subjected to cyclic shear in laboratory tests. 
THE CONTENTS OF THIS REPORT ARE NOT TO BE USED FOR ADVERTISING, PUBLICATION, OR PROMOTIONAL PURPOSES. CITATION OF TRADE NAMES DOES NOT CONSTITUTE AN OFFICIAL ENDORSEMENT OR APPROVAL OF THE USE OF SUCH COMMERCIAL PRODUCTS. 
This report was prepared by Professors Zdenek P. Bazant and Raymond J. Krizek of Northwestern University under Contract No. DACW3975-M-3739 as part of ongoing work at the U. S. Army Engineer Waterways Experiment Station (WES) under CWIS 31145 work unit entitled, "Liquefaction Potential of Earth Dams and Foundations."

The contract was monitored by Dr. William F. Marcuson III of the Earthquake Engineering and Vibrations Division (EE\&VD), Soils and Pavements Laboratory (S\&PL). General guidance was provided by Mr. James P. Sale, Chief of S\&PL; Mr. Stanley J. Johnson, Special Assistant to the Chief of S\&PL; and Mr. Walter C. Sherman, Jr., and Dr. Fancis G. McLean, former Chief and Chief of EE\&VD, respectively.

Contracting officer wasCOL G. H. Hilt, CE, Director of WES. 
Preface . . . . . . . . . . . . . . . . . . . . 2

Introduction ........................... 4

Rearrangement Measure .. . . . . . . . . . . . . . 5

Densification Due to Deviatoric Strains ............ 7

Intrinsic Time .................... . 11

Endochronic Stress-Strain Law for Sand . . . . . . . . . 12

Liquefaction of Undrained Sand . . . . . . . . . . . 18

Conclusions . . . . . . . . . . . . . ......... 23

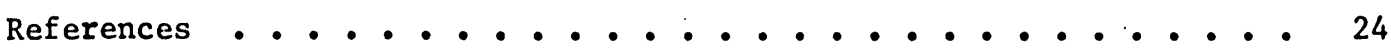




\section{INTRODUCTION}

Mathematical analyses involving the liquefaction of sand deposits require a realistic constitutive law for the nonlinear bchavior of sand, especially under conditions of cyclic shear. Although considerable effort has been devoted to the development of constitutive laws based on theories of plasticity and hypoelasticity (Coon and Evans, 1971; Lade and Duncan, 1975), the success of these theories has been quite limited, and no constitutive law is presently available to adequately describe the densification and hysteresis of sand subjected to cyclic shear. Practical studies have led to the establishment of simplified empirical stressstrain relationships in which the material properties (such as shear modulus, damping coefficient, densification parameter, etc.) depend on the number of cycles. However, this variable may not appear in any general constitutive law that is to be applied to other than sinusoidal load histories, such as those typical of earthquakes. Also, these formulations are not in a form that would be applicable to multi-dimensional situations and general drainage conditions.

Developed herein is an entirely new type of constisutive law that achieves the aforementioned objectives. This law may be termed "endochronic" because the nonlinearity of the material is introduced chiefly by means of an intrinsic time parameter, which is an independent scalar variable whose increments depend, in general, on both time increments and deformation increments. The dependence on time increments disappear, of course, in the case of time-independent inelastic behavior, as is the case with sand. In this case the intrinsic time parameter represents the length of the path traced by the states of the material in a sixdimensional space (with a general metric tensor) whose coordinates are 
the strain components. The length of this path constitutes a natural choice for the independent variable, and it has been introduced in many theoretical works (Bazant and Bhat, 1974). However, the usefulness of this path parameter for the practical description of inelastic behavior was apparently not realized until Valanis (1971) employed it for metals to develop a stress-strain law that correctly characterizes strainhardening, unloading behavior, the effect of pretwist on axial behavior, the contraction of hysteresis loops with cyclic straining, and the strain rate effect. From the work of Valanis it became apparent that endochronic theory provides a very fruitful approach to describing materials in which inelastic strain develops gradually.

Using Valanis' theory as a point of departure, a method of extending endochronic theory to concrete, rock, and sand has been proposed in general terms by Bazant $(1974 \mathrm{~b})$, and the detailed formulation for concrete has been given by Bazant and Bhat (1974). This extension has required the introduction of several novel concepts to handle (a) the sensitivity of intrinsic time to hydrostatic pressure, (b) inelastic dilatancy (or densification) due to shear straining, and (c) the strain-softening tendency. The purpose of this work is to present the detailed development of endochronic theory for sand and to show how this theory can be used in the analysis of seismic liquefaction.

\section{REARRANGEMENT MEASURE}

The source of inelasticity in sand is the irreversible rearrangement of grain configurations associated with deviatoric strains. Thus, it is convenient to characterize the accumulation of rearrangement by an appropriate variable $\xi$, termed the rearrangement measure, which will be used as the independent variable in the stress-strain law. For this 
reason and also to ensure that the energy dissipation rate is always positive, 5 may never decrease. Since the rearrangement of grain configurations results froin deformation, $d \xi$ is a function of the strain increments $d \varepsilon_{i j}$. Assuming that the development of inelastic strain is gradual, $d \xi$ must be a continuous and smooth function of $d \varepsilon_{i j}$, and $(d \xi)^{s}$ with certain exponents may be expanded in a tensorial power series as $(d \xi)^{s}=p^{(0)}+p_{i j}^{(1)} d \epsilon_{i j}+p_{i j k 1}^{(2)} d \varepsilon_{i j} d \epsilon_{k 1}+p_{i j k 1 m n} d \varepsilon_{i j} d \epsilon_{k 1} d \epsilon_{m n}$

in which latin indices refer to cartesian coordinates $x_{i}(i=1,2,3)$

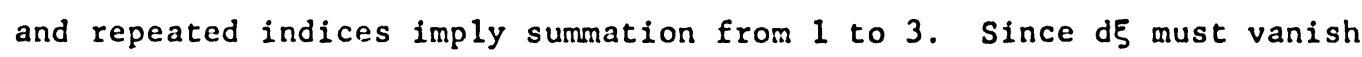
as $d \varepsilon_{i j} \rightarrow 0$, it is necessary that $p(0)=0$. Furthermore, the condition that the energy dissipation rate must be always positive requires that $p_{i j}(1)=0$ and that the coefficients $p_{i j k l}(2)$ define a positive definite quadratic form. Because $d \varepsilon_{i j}$ is infinitesimal, terms of order higher than 2 are negligible; accordingly, $p_{i j k l m n}+\ldots=0$. Finally, it is necessary to meet the condition of isotropy and the condition that $d \xi=0$ for purely volumetric strains, because no rearrangement of grain configurations takes place in this case. By use of the same reasoning as that applied in developing endochronic theory for concrete (Bažant and Bhat, 1974 Eqs. 2 to 5), it can be shown that the only expression satisfying the latter two conditions is

$$
d \xi=\sqrt{J_{2}(d \xi)}=\sqrt{\frac{1}{2} d e_{i j} d e_{i j}}
$$

in which $J_{2}(\ldots)$. is the second invariant of the deviator of the tensor in parenthesis, $\underset{\sim}{\varepsilon}=\left[\varepsilon_{i j}\right]$ is the strain tensor, $e_{i j}=\varepsilon_{i j}-\delta_{i j} \varepsilon_{k k} / 3$ is the deviator of the tensor $\varepsilon_{1 j}$ and $\delta_{1 j}$ designates Kronecker delta. A multiplicative constant is omitted in Equation 2 because d $\xi$ will be multiplied in the stress-strain law by an arbitrary constant. In the special case of pure shear strain, $\gamma=2 \varepsilon_{12}$ and Eq. 2 ylelds

$$
d \xi=\frac{1}{8}|d \gamma|
$$


More specifically, for the case of cyclic pure shear in which $\gamma=\gamma_{0}$ sin wt, Eq. 3 yields $\Delta \xi=\gamma_{0} / 2$ per cycle, so that at the end of the $N$-th cycle $\xi$ may be expressed as

$$
\xi=\frac{1}{2} Y_{0} N=\frac{1}{2} \gamma_{0} \frac{\omega}{\pi} t
$$

Hence, $\xi$ is a measure of the number of cycles in the loading history.

\section{DENSIFICATION DUE TO DEVIATORIC STRAINS}

The densification of sand may be characterized by the volumetric strain $\lambda$ whose sign is chosen to be negative when the volume decreases. If vertical accelerations are not large enough to cause any significant separation or jumping of particles (as is the case in many earthquakes), the densification is produced almost exclusively by interparticle slips that result in a rearrangement of grain configurations. Subject to this restriction, $d \lambda$ must be proportional to $d \xi$, and the dependence of the densification increment per cycle of shear on the strain amplitude and the number of cycles may be expressed by

$$
\mathrm{d} \lambda=-\frac{\mathrm{d} x}{\mathrm{c}(x)} ; \quad \mathrm{d} x=\mathrm{c}(\underset{\varepsilon}{\sigma}, \sigma) \mathrm{d} \xi
$$

The function $c(x)$ models the decrease in the densification increments per cycle with an increase in the number of cycles $N$. Accordingly, $c$ is a continuous, monotonically increasing, positive function, which may be termed the densification - hardening function. Eq. 5 may be integrated to yield $\lambda=\lambda(x)$ and inverted to give $x=x(\lambda)$; because $c(x)$ is always positive, the simplest choice for $c$ is the linear expression

$$
c(x)=c_{0}(1+\alpha x)
$$

for which

$$
\lambda=-\frac{1}{c_{0} \alpha} \ln (1+\alpha x)
$$


in which $\alpha$ and $c_{0}$ are constants for a given sand. Integration is also easy for the more general function $c \sim(1+\alpha x / k)^{k}$. The function $c$ may, in general, introduce a dependence of densification rate on volumetric stress, $\sigma$; however, for the sand studied by Silver and Seed (1971) no dependence on $\sigma$ was discerned, and thus $C$ may be considered independent of $\sigma$ (at least for some sands). The function $:(\varepsilon, \sigma)$, which may be called the densification-softening function, models the increase in densification rate with increasing strain amplitude $\gamma_{0}$ in cyclic shear. Because of isotropy, only the invariants of the strain tensor $\underset{\varepsilon}{\sim}$ may appear in $C$. The third invariant $I_{3}(\underset{\sim}{\sim})$ may be neglected because it is third-order small when strains are small; however, even if it were not neglected for this reason, $I_{3}(\underset{\sim}{\epsilon})=0$ in the case of pure shear. The first invariant $I_{1}(\varepsilon)$ need not be considered because the dependence on $I_{1}(\varepsilon)$ is equivalent to the dependence on relative density $D_{r}$ which will be considered separateiy. Thus, $C$ may be regarded as a function of only $\mathrm{J}_{2}(\varepsilon)$, the second invariant of the deviator of $\underset{\sim}{\epsilon}$, and one suitable choice for $\mathrm{C}$ is

$$
C(\varepsilon)=2 q\left[8 J_{2}(\varepsilon)\right]^{(q-1) / 2}
$$

in which $q$ is a non-negative constant for a given sand. In the special case of pure shear, Eq. 8 becomes $C \underset{\sim}{(\epsilon)}=2 q|\gamma|^{q-1}$ and Eq. 5 yields $\mathrm{d} x=\frac{3}{4} q|\gamma|^{q-1}|d \gamma|$. For cyclic shear the increment of $x$ over the cycle is $4\left(\frac{3}{4} \gamma_{0}^{q}\right)$, where $\gamma_{0}$ is the amplitude of shear strain. Thus, after $N$ cycles $x=\gamma_{0}^{q} N$, and Eq. 7 becomes

$$
\lambda=-\frac{1}{c_{0} \alpha} \ln \left(1+\alpha \gamma_{0}^{q} N\right)
$$

To illustrate the ability of this theory to describe experimental results, Eq. 9 has been fitted to test data reported by seed and Silver (1971) for the densification of a given sand at a relative density $D_{r}$ of $45 \%$ under cyclic shear, and a typical fit is shown in Figure 1. 


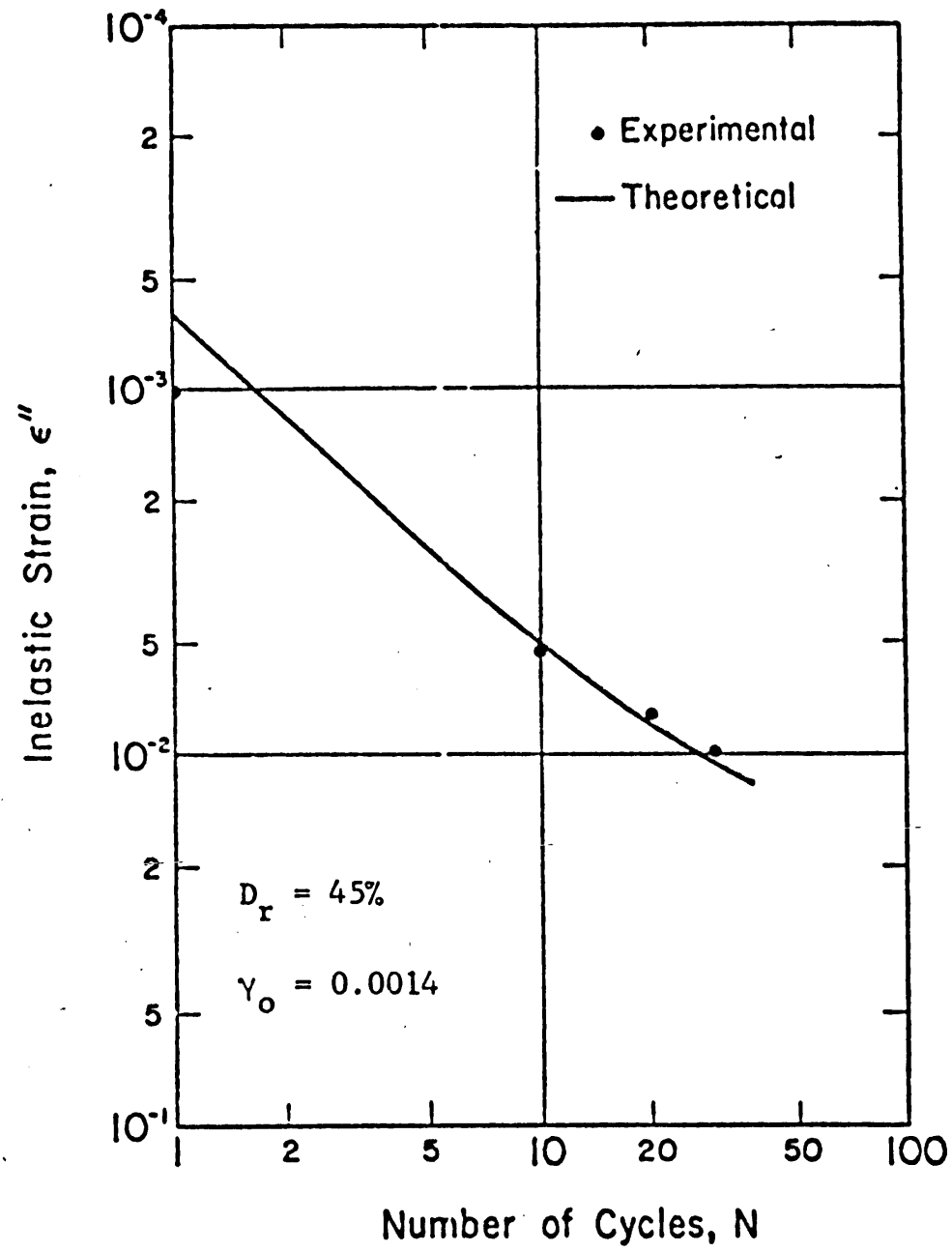

Figure 1. Inelastic Strain versus Number of Cycles

(for the case illustrated the values of the material parameters are $q=1.4, \alpha=1.0$, and $c_{0}=1.0$ ). The material parameters $\alpha, q$, and $c_{0}$ will, in general, depend on the relative density $D_{r}$ :

$$
\alpha=\alpha\left(D_{r}\right) ; q=q\left(D_{r}\right) ; c_{0}=c_{0}\left(D_{r}\right)
$$

Although the nature of this dependence requires further investigation, it may be concluded even without quantitative evaluation by use of test data, that $c_{0}\left(D_{r}\right)$ must approach zero as $D_{r}$ approaches a certain critical density $D_{r}^{C r}$, and it must become negative when $D_{r}>D_{r}^{c r}$ to reflect the fact that shear at overcritical densities causes an increase in volume (called 
dilatancy) rather than densification. One suitable function may be

$$
c_{0}= \pm C_{0}\left|1-D_{r} / D_{r}^{c r}\right|^{r}
$$

in which $C_{0}$ and $r$ are constants for all densities of a given sand, but may perhaps depend on confining stress. The critical density $D_{r}^{c r}$ for a given sand will also depend on the confining stress and possibly other variables (e.g. $\mathrm{J}_{2}\left(\underset{\sim}{(\varepsilon))}\right.$ ). For $\mathrm{D}_{\mathrm{r}}<\mathrm{D}_{\mathrm{r}}^{\mathrm{cr}}$ the relative density gradually increases due to shear straining.

Because the dependence of $D_{r}$ on $\lambda$ would considerably complicate the analysis, $D_{r}$ must be approximated by the constant $D_{r}{ }^{0}$. In addition, this densification law will probably not be too accurate for describing the detailed progress of densification within a given cycle. For example, the application of a small increment of shear strain within a cycle may cause a dense sand to dilate, even though the repeated application of many cycles of shear strain may produce a negative cumulative volume change or densification. This phenomenon cannot be modeled by this theory unless $c_{0}$ is considered to be a function of $\underset{\sim}{\varepsilon}$. However, for most practical applications the detailed progress of densification within a given cycle is not of major concern. In general, the dependence of $D_{r}$ on $\lambda$ is not too important, because the change in $D_{r}$ that is normally produced by shear straining alone usually does not exceed about $5 \%$ (based on the assumptions that the relative density range from 0 to $100 \%$ corresponds to approximately a $15 \%$ change in the actual density of sand and that shear straining alone generally does not cause volumetric strains of more than about $1 \%$ ). The foregoing formulation of the densification law is analogous to that employed in characterizing the dilatancy of concrete (Bazant and Bhat, 1974; Bazant, 1974b); the only difference consists in the form of the functions $C(\varepsilon)$ and and $c(x)$. However, with regard to metals (Valanis, 1971) the inclusion of dilatancy or densification represents one of the major extensions needed. 


\section{INTRINSIC TIME}

Since the increments of irreversible (inelastic) strains in sand are caused by interparticle slips, they must be proportional to increments of the rearrangement measure $\xi$ and the proportionality coefficient may, in general, depend on the state of strain and stress. To express this fact a new independent variable $\eta$ may be introduced such that on $=F(\underset{\sim}{\sigma}, \sigma) d \xi$. As in the case of densification, the increment of inelastic strain per cycle diminishes as the number of cycles increases. This is true because the contacts with peak interparticle shear stresses, which are the potential locations of slips, gradually become exhausted as the inelastic strain accumulates. To account for this effect, it is expedient to introduce a new independent variable $\zeta$, termed intrinsic time, whose growth relative to $\eta$ or $\xi$ gradually diminishes. This phenomenon may be described by the relations

$$
\mathrm{d} \zeta=\frac{\mathrm{d} \eta}{\mathrm{f}(\eta)} ; \quad \mathrm{d} \eta=\mathrm{F}(\underset{\sim}{\epsilon}, \sigma) \mathrm{d} \xi
$$

in which $f(\eta)$, which may be termed a strain-hardening function, is a continuously increasing positive function of $\eta$. Since $d \eta$ is multiplied by an arbitrary constant $z_{1}$ in the stress-strain relation, $f(0)$ can be set equal to unity. One simple choice for $f(\eta)$ is

$$
f(\eta)=\left(1+\frac{B}{r} \eta\right)^{r}
$$

in which $B$ and $r$ are constants for a given sand. When $\eta$ is sufficiently small, $f(\eta) \approx 1+\beta \eta$ for any value of $r$. Since $f(\eta)$ is always positive, Eq. 12 may be integrated to yield $\zeta=\zeta(\eta)$ and inverted to yield $\eta=\eta(\zeta)$. With the use of Eq. 13, Eq. 12 gives $\zeta=(1+\beta \eta)^{1-r} \beta r /(1-r)$ for $r \neq 1$ and $(1 / \beta) \ln (1+\beta \eta)$ for $r=1$. 
The function $F$, which may involve only the invariants of $\underset{\sim}{\sim}$ and $\underset{\sim}{\sigma}$, accounts for the softening in the stress-strain relation of the material on approaching the peak (maximum) values of stress (Bazant, 1974a). Therefore, $F$ may be termed a strain-softening function. However, within the range of strains commonly encountered in seismic loadings, it appears that $F$ may be set equal to unity as an acceptable approximation.

\section{ENDCCHRONIC STRESS-STRAIN LAW FOR SAND}

As a consequence of material isotropy, the quasi-linear incremental stress-strain relation may be separated into one relation for the volumetric components and one relation for the deviatoric components. In the most general and plausible form of the deviatoric stress-strain relation, $s_{f_{j}}$ : may be regarded as a functional of the past history of the stress deviator $e_{i j}$. The basic assumption and most advantageous feature of the endochronic theory is that this functional may be expressed as a linear functional in terms of the intrinsic time $\zeta$ because the nonlinearities are assumed to be incorporated in $\zeta$ (as well as $\lambda$ ). This approach is much simpler than formulating nonlinearities by means of incremental or tangential moduli, as in hypoelasticity, because these latter moduli form a fourth-rank tensor while $\zeta$ is a scalar. The linear functional may be written as

$$
s_{i j}(t)=2 \int_{0}^{t} \bar{G}\left(t-t^{\prime}\right) \operatorname{de}_{i j}\left(t^{\prime}\right)
$$

in which the integral is a stieltjes integral and the hereditary kernel $\bar{G}$ describes the material properties. It is well known (Bažant, 1974a) that any reasonable hereditary kernel can be approximated to any desired accuracy by a series of decaying real exponentials (Dirichlet series). Since $\zeta$ is the independent variable in which the stress-strain relation 
becomes quasi-linear, the sum may be written as

$$
\bar{G}\left(t-t^{\prime}\right)=\sum_{\mu=1}^{n} G_{\mu} e^{-\left(\zeta(t)-\zeta\left(t^{\prime}\right)\right) / Z_{\mu}}
$$

in which $z_{\mu}$ are chosen constants; one suitable choice for $z_{\mu}$ is $z_{1} 10^{\mu-1}$ (i.e., one $z_{\mu}$ is chosen for each order of magnitude of $\zeta$ ); all orders of magnitude of $\zeta$ that are of interest must be covered. Upon substitution from Eq. 15, Eq. 14 may be cast in the form -

$$
s_{i j}=\sum_{\mu} s_{i j_{\mu}} ; s_{i j}=2 G_{\mu} e^{-\zeta(t) / z_{\mu}} \int_{0}^{t} e^{\zeta\left(t^{\prime}\right) / z_{\mu}} d e_{i j}\left(t^{\prime}\right)
$$

in which $s_{i j_{\mu}}$ are hidden stresses (internal variables). Letting de $_{i j}=$ $\left(d e_{i j} / d \zeta_{\mu}\right)\left(d \zeta_{\mu} / d t^{\prime}\right) d t^{\prime}$, it may be verified that the variables $s_{i j_{\mu}}$ satisfy the following differential equations:

$$
2 G_{\mu} d e_{i j}=d s_{i j_{\mu}}+s_{i j_{\mu}} \frac{d \zeta}{z_{\mu}} ; s_{i j}=\sum_{\mu} s_{i j}
$$

which represent the deviatoric stress-strain relations. The structure of Eq. 17 may be visualized as a generalized Maxwell model with spring moduli $G_{\mu}$, dashpot viscosities $G_{\mu} Z_{\beta}$ relative to intrinsic time $\zeta$ (rather than actual time $t$ ), and relaxation times $z_{\mu}$ on the intrinsic time scale. Because of the limited range of $\zeta$-values that are of practical interest, a single exponential term of Eq. 15 ought to suffice in most cases. Then, $s_{i j_{\mu}} \equiv s_{i j}$ and the deviatoric stress-strain relation given by Eq. 17 reduces to

$$
d e_{i j}=\frac{d s_{i j}}{2 G}+d e_{i j}^{\prime \prime}, d e_{i j}^{\prime \prime}=\frac{s_{i j}}{2 G} \frac{d \zeta}{Z_{1}}
$$

in which $G$ is the shear modulus and $e_{i j}^{\prime \prime}$ is the inelastic strain. Eq. 18 is similar to the constitutive relation for a single Maxwell unit.

The volumetric stress-strain relation cannot contain any inelastic volumetric strains that are dependent on volumetric stress, because 
purely volumetric deformations are assumed to be fully reversible. Therefore the only possible volumetric inelastic strain $\varepsilon^{\prime \prime}$ is the densification $\lambda$; if thermal strain were considered, $d e^{\prime \prime}=d \lambda+\alpha d T$. Thus, the volumetric stress-strain relation at constant temperature is

$$
d \epsilon=\frac{d \sigma}{3 K}+d \lambda
$$

in which $K=$ bulk modulus of sand. The endochronic stress-strain law given by Eqs. 18 and 19 is analogous to that verified for concrete (Bazant and Bhat, 1974), and it models in a particularly simple way (without any inequalities) the irreversibility upon unloading and the hysteretic behavior of sand subjected to strain cycles. Irreversibility upon unloading, which represents the salient aspect of all inelasticity, may be illustrated by writing Eq. 18 for pure shear $\left(\tau=s_{12} ; \gamma=2 \varepsilon_{12}\right)$ in the form $\Delta T=G \Delta Y-G \Delta \gamma^{\prime \prime}$, in which $G \Delta Y$ is the elastic stress increment and $\mathrm{G} \Delta \mathrm{Y}^{\prime \prime}$ is the inelastic stress increment; these increments for a prescribed value of $\Delta y$ are depicted in Figure 2.

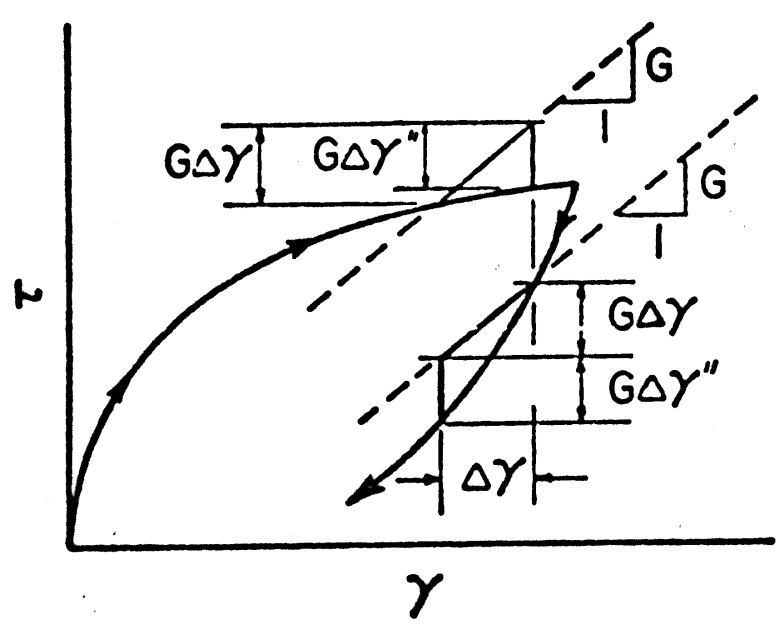

Figure 2. Elastic and Inelastic Stress Increments 
At the point where loading suddenly reverses to unloading, the sign of $G \Delta Y$ becomes negative, but $G \Delta \gamma^{\prime \prime}$ does not change because $d \zeta$ and $d \xi=|d \gamma| / 8$ do not change sign. These increments for unloading are also 11 lustrated in Fig. 2, from which it is clear that that unloading branch is much steeper than the loading branch. Studies of cyclic shear test data have indicated that Eqs. 18 and 19 are capable of describing experimental results quite well within a limited range of amplitude for the shear angle (about $10^{-4}$ to $5 \times 10^{-4}$ ), but the quantitative agreement is not as good for a broader range of amplitudes.

To extend the range of applicability, Eq. 17 has been considered with $n=2(\mu=1,2), Z_{1}<\infty, Z_{2} \rightarrow \infty, G_{1}>0$, and $G_{2}>0$; the resulting expression, which corresponds to the stress-strain relation for a standard solid model in intrinsic time, can still be reduced to a single first-order differential equation (without hidden stresses). Although the inelastic strain expression, $\Delta \gamma^{\prime \prime}=\left(\tau-G_{2} \gamma\right) \Delta \zeta / G Z_{1}$ in this case decreases the slopes of the unloading branches at higher values of $\gamma$ and allows somewhat improved fits in the first cycle, this model is not able to adequately describe the data over the full range of strain history because after a few cycles it yields hysteresis loops that are too narrow. One obvious improvement is to take $\mathrm{Z}_{2}<\infty$ and add a third Maxwell unit; however, this approach is not very convenient because hidden stresses $s_{i j_{j}}$ must be introduced. As a simple expediency to resolve this problem and satisfactorily characterize all data, the shear modulus $G$ may be considered a variable, rather than a constant (as implied in Eqs. 14 to 16). Specifically, $G$ may be assumed to be a function of the maximum value of $\mathrm{J}_{2}(\varepsilon)$ that the sand has experienced up to the current time $t$; Eqs. 14 to 16 require generalization in this case. 
The manifested stiffening of sand with increasing hydrostatic pressure $-\sigma$ is properly modeled by considering $G$ to be a function of $-\sigma$. This stiffening may be explained by the fact that an increase in hydrostatic (confining) pressure prevents slip at some of the intergranular contacts due to higher normal forces, so that only elastic deformation of the grains is possible at these contacts and the microstructure becomes stiffer as a result.

Since the contact areas between grains increase with the confining stress, the solid skeleton becomes stiffer and fewer grain contacts slip as the confining stress is increased. Based on extensive experimental data for the dynamic shear modulus of sand, $G$ has been shown to be essentially proportional to the square root of the confining stress (Richart, Hall, and Woods, 1970). Accordingly, the shear modulus $G$ may be expressed as

$$
G=\sqrt{-M \sigma}
$$

in which $M$ is a material parameter that has been taken as $7060 \mathrm{MN} / \mathrm{m}^{2}$ to obtain the fit of test data shown in Figure 3 . These data, provided by
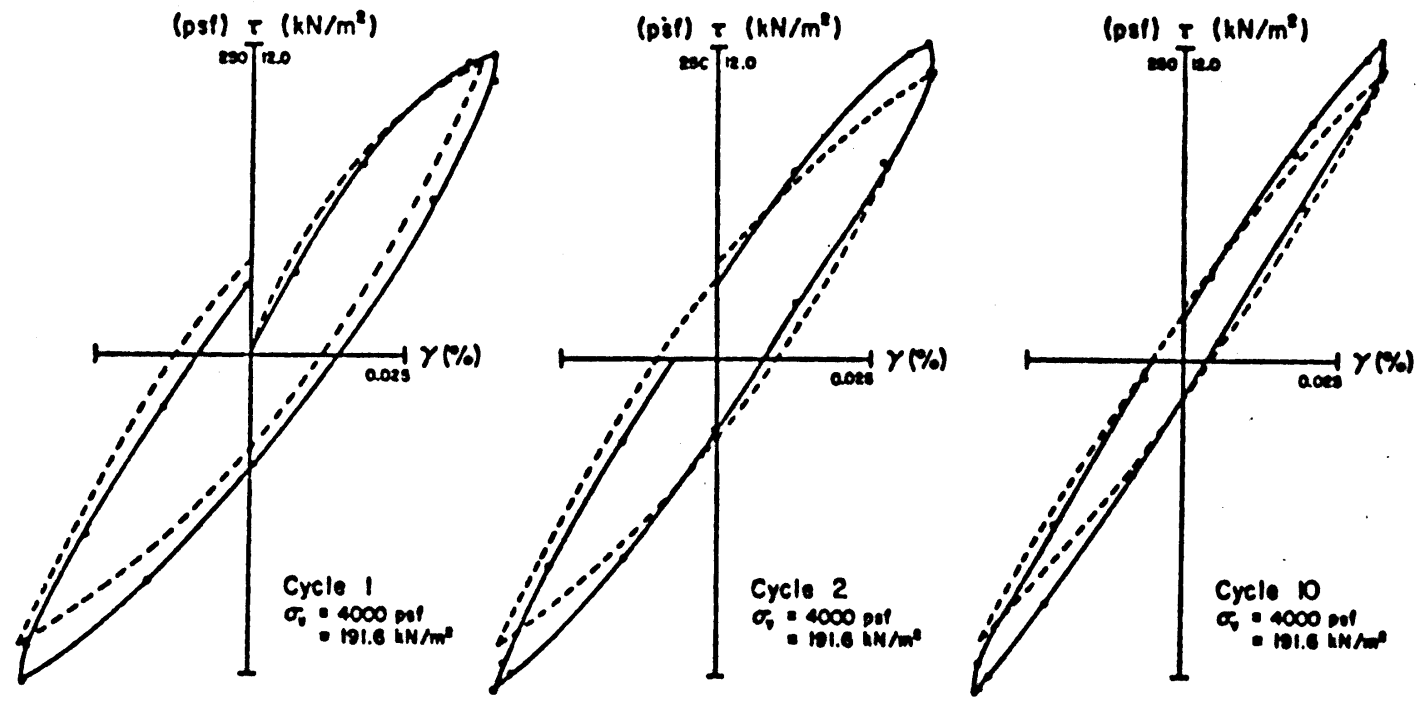

F1gure 3. Hysteresis and Stiffening of Sand under Cyclic Shear 
Silver (1974), pertain to cyclic shear tests on a sand at a relative density of $60 \%$ and a vertical effective stress of 4000 psf $\left(191.5 \mathrm{kN} / \mathrm{m}^{2}\right)$. It is seen that the theory correctly models both the increase in peakstrain secant (dynamic) modulus and the decrease in the area of the hysteresis loop (which is a measure of damping) as the number of cycles increases. In addition, the peak-strain secant modulus obtained from the theory can be shown to decrease as the strain amplitude increases.

From the analysis of more extensive data, however, it appears that $M$ may be considered constant only when the range of shear strain amplitude is not too broad (definitely not exceeding one order of magnitude). For a broader range $M$ must be considered dependent on the strain amplitude, and because the strain amplitude may not appear in a general constitutive equation, $M$ must be a function of the strain history. Although such a strain history dependence has been implicd in work reported by Finn, Bransby, and Pickering (1970), there is no clear physical microstructural explanation for such an effect and it may be viewed as somewhat of an artifice, especially in the context of endochronic theory (this artifice implies that $G$ possesses a memory property, whereas the inelastic strain is formulated without recourse to memory). Furthermore, for a given sand at different values of relative density, the material parameters will, in general, be functions of $D_{r}$. When more extensive data become available, it is probable that $F(\underset{\sim}{\epsilon}, \underset{\sim}{)}$ ) in Eq. 12 will manifest a dependence on $\sigma$ (perhaps similar to that found for concrete (Bazant and Bhat, 1974), rather than the assumption of $\mathrm{F}(\underset{\sim}{\tau} \underset{\sim}{\sigma}=1$, as used herein), because hydrostatic pressure should affect both the stiffness of the microstructure and the extent of particle slippage within the microstructure. It may be possible to replace the 
dependence of $G$ on $\underset{\sim}{\varepsilon}(t)$ with a dependence of $F$ on $p$ and $J_{2}(\varepsilon)$, combined with the use of several Maxwell units in the chain. This question could be resolved by using the theory to analyze test data for small (or large) strain amplitudes followed by large (or small) strain amplitudes, or for asymmetric cycles; however, no such data are presently available. The stress-strain law given by Eqs. 18 and 19 is analogous to that verified previously for concrete (Bažant and Bhat, 1974); the only differences consist in the form of the functions $F$ and $f$ and the variation of $G$. The hydrostatic pressure sensitivity is a common feature with concrete and one of the major differences with regard to metals.

\section{LIQUEFACTION OF UNDRAINED SAND}

When a loose saturated sand under constant total stress is subjected to cyclic deviatoric deformations, the resulting densification causes a compression of the pore water and a build-up of the pore vater pressure, which, in turn, causes a corresponding decrease in the effective (intergranular) stress, because the total vertical normal stress in the combined solid-water system must remain constant due to a constant overburden. This decrease in the effective stress is associated with a loss of friction between the grains and a decrease in the shear modulus. In the extreme case, when the effective stress becomes zero, all friction is lost and the shear modulus is zero. Hence, the sand behaves as a liquid.

To express the interaction of sand and water, it is necessary to treat the saturated sand as a two-phase porous medium. The constitutive equation for a two-phase medium has been rigorously developed by Biot (1956, 1957) for the case of elastic behavior, and an extension including inelastic densification has been presented by Bazant and Krizek (1975). The deviatoric 
stress-strain relation for the inelastic isotropic two-phase medium has the same form as that of the solid phase alone (i.e., dry sand) but the volumetric stress-strain relation is different and may be written as (see Eq. 45, Bažant and Krizek, 1975):

$$
\begin{aligned}
& \mathrm{d} \sigma=P d \epsilon+Q d \epsilon_{F}-\left(P-\frac{1-n}{n} Q\right) d \epsilon^{\prime \prime} \\
& d \sigma_{F}=Q d \epsilon+R d \epsilon_{F}-\left(Q-\frac{1-n}{n} R\right) d \epsilon^{\prime \prime}
\end{aligned}
$$

in which $\sigma$ is the portion of the total volumetric (hydrostatic) stress $\sigma_{t}$ carried by the solid phase; $\sigma_{F}$ is the remaining portion of $\sigma_{t}$ carried by the fluid phase (i.e. $\sigma_{F}=\sigma_{t}-\sigma=\sigma_{t}+n p_{w}$ ); $n$ is the porosity; $p_{w}$ is the pore water pressure; $\epsilon$ and $\varepsilon_{F}$, defined as $\varepsilon=\operatorname{div} \underset{\sim}{u}$ and $\varepsilon_{F}=\operatorname{div} u_{\mathbb{E}}$, are the volumetric strains of the solid and fluid phases, respectively; $\underset{\sim}{u}$ and $\underset{\sim F}{u}$ are the displacements of the solid phase and the fluid phase; $P, Q$, and $R$ are the incremental (tangent) elastic moduli of the two-phase medium; and $\epsilon^{\prime \prime}$ is the inelastic volumetric strain in the solid phase.

In an approximate analysis of seismic liquefaction, the saturated sand may be assumed to be undrained, provided a large region of sand remains in a homogeneous state. Very often, however, this condition may not exist, since very small displacements of water can cause a large change in pressure due to the small compressibility of water. This may be important even if the duration of an earthquake is too short to allow any significant flow of water from the sand. Furthermore, because the overburden of any element is constant, the total volumetric stress $\sigma_{t}$ in the two-phase medium may be considered constant, provided the ratio of lateral to vertical stress also remains constant and provided the vertical dynamic stress (whose time average is zero) is neglected. For the case where $d \sigma_{t}=0$, the increment of pore water pressure due to a densification increment is (Bazant and Krizek, 1975; Eq. 48) 


$$
d p_{w}=-\frac{d \varepsilon^{\prime \prime}}{c_{d}} \approx-\frac{d \varepsilon^{\prime \prime}}{c_{b}}
$$

in which $d e^{\prime \prime}$ is the inelastic volumetric strain and $C_{d}$ is the densification compliance, which is given (Bažant and Krizek, 1975; Eq. 49) by $n^{2} /\left\{R-(Q+R)^{2} /\right.$ $(P+2 Q+R)\}$. For typical sands in a loose or dense state, $C_{d}$ is approximateiy equal (with an error of less than a few percent) to the bl:lk compressibility of drained sand $C_{b}$, (Bažant and Krizek, 1975; Eq. 50) $C_{b}$ is defined as $\partial \epsilon / \partial \sigma_{t}$ at constant $P_{w}$, and it is equal to $R /\left(P R-Q^{2}\right)$. It must be emphasized that $C_{d}$ does not equal the bulk compressibility of undrained sand $C_{t}=$ $(P+2 Q+R)^{2}$, but it is orders of magnitude larger (Bazant and Krizek, 1975). Since the total stress $\sigma_{t}$ is constant, the change in the effective (intergranular) stress $\sigma^{\prime}$ at constant total stress $\sigma_{t}$ is

$$
d \sigma^{\prime}=d p_{w}
$$

The combination of Eqs. 22,23 , and 5 (with $d \varepsilon^{\prime \prime}=\mathrm{d} \lambda$ ) for an undrained sand at constant total volumetric stress yields

$$
d \sigma^{\prime}=-\frac{d \lambda}{C_{d}}=-\frac{d x}{C_{d} c(x)}
$$

which states that the solid phase of a saturated sand densifies according to the same law as the dry sand,provided the effective (intergranular) stress is the same in both cases. Although the difference in friction between wet and dry grain contacts may cause some deviation from this assumption, this is probably insignificant; for example, Richart, Hall, rand Woods (1970, p. 156) indicated no appreciable difference in the shear wave velocity (and hence the shear modulus) for dry, drained, and saturated Ottawa 20-30 sand, and it is reasonable to expect that the friction between grains would behave similarly. Another possible deviation may be due to viscous and inertia forces opposing the relative movements of grains; however, these movements are only microscopic, since the average 
macroscopic relative movement of water and sand in the undrained condition is zero. Furthermore, it should be realized that the deviator strains produce only the possibility of densification, whereas the driving force is provided by the volumetric effective stress $\sigma^{\prime}$. This is supported by the fact that there should be no densification when $\sigma^{\prime}=0$. Therefore, the effective stress serves the purpose of overcoming the microscopic viscous and inertia forces. It is thus conceivable that the densification rate of a saturated sand may depend on $\sigma^{\prime}$, even though the effect of $\sigma^{\prime}$ is negligible in a dry sand. Accordingly, it might be appropriate to introduce a correction coefficient $L\left(\sigma^{\prime}\right)$ into Eq. 24 , as follows:

$$
d \sigma^{\prime}=-L\left(\sigma^{\prime}\right) \frac{d \lambda}{C_{d}}
$$

On the other hand, there is no reason to include the pore pressure $p_{w}$ or the total volumetric stress $\sigma_{t}=\sigma^{\prime}-p_{w}$ in this equation. As an alternative to the dependence of $L$ on $\sigma^{\prime}$, the effect of $\sigma^{\prime}$ on the build-up of $\mathrm{p}_{\mathrm{w}}$ may also stem from the dependence of $\mathrm{C}$ (Eq. 5) on $\sigma^{\prime}$. Although no such dependence was discerned for one type of sands (Silver and Seed, 1971), 1t may be significant for other types of sand.

Consider now a saturated sand specimen that is always in a homogeneous state of stress and strain (for laboratory test conditions this assumption is extremely difficult, if not impossible, to satisfy due to local disturbances produced by the loading device) while being subjected to a sinusoidal history of shear stress $T=T_{0}$ sin wt under constant volumetric stress $\sigma$ all other stress components being zero. The tendency for liquefaction of this specimen may be assessed by integrating Eq. 27 in time and combining the relations

$$
d \gamma=\frac{1}{G(\sigma, \gamma)}\left(d \tau+\tau \frac{d_{0}^{r}}{z_{1}}\right)
$$




$$
\begin{gathered}
d \zeta=\frac{d \xi}{\left(1+\frac{\beta}{r} \xi\right)^{r}} ; d \xi=\frac{1}{8}|d \gamma| \\
d \lambda=-\frac{d x}{c_{0}(1+\alpha x)} ; d x=\frac{1}{4} q|\gamma|^{q-1}|d \gamma|,
\end{gathered}
$$

which stem from Eqs. 3, 6, 8, 12, 13, 18, and 19; G is given by Eq. 20 . The numerical step-by-step integration of Eqs. 25 to 28 was accomplished by using a computational algorithm of the same type as that described by Bazant and Bhat (1974); the calculation of each time step is interated several times, first using estimated and then improved values of $\xi, \zeta$, and $x$.

This theory was applied to liquefaction data reported by Finn, Branshy, and Pickering (1970) for a test conducted on a sample of loose Ottawa sand. The specimen was loaded by a controlled cyclic shear stress of amplitude $0.0245 \mathrm{~N} / \mathrm{mm}^{2}$, and the resulting shear strains were greater than 0.001 . Since hysteresis loops and the densification response for the sand in a dry state were not reported, material parameters were selected on the basis of test data (Silver, 1974) for a sand with similar grain characteristics and $\gamma_{0}=0.0014$; only the coefficient $L$ was considered arbitrary, and the best fit of test data was obtained with $L=1.7\left(\sigma_{0}+\sigma^{\prime}\right)$, where $\sigma_{0}=0.196 \mathrm{~N} / \mathrm{mm}^{2}$ and $\sigma^{\prime}$ is negative for compression (L increases as the effective stress decreases with the progress of the test). Figure 4 shows a good agreement between experimental and theoretical results. The sharp increase in the slope of the $\mathrm{p}_{\mathrm{w}}-\mathrm{N}$ diagram is due to the decrease. in the shear modulus as the effective stresses decreases. As a consequence of the reduced shear modulus, the amplitudes of the shear strain increase, and this, in turn, causes a faster accumulation of $\xi$, a faster densification, and a more rapid drop in the shear modulus. This behavior is a type of feedback that is characteristic of instabilities of motion, in general. 


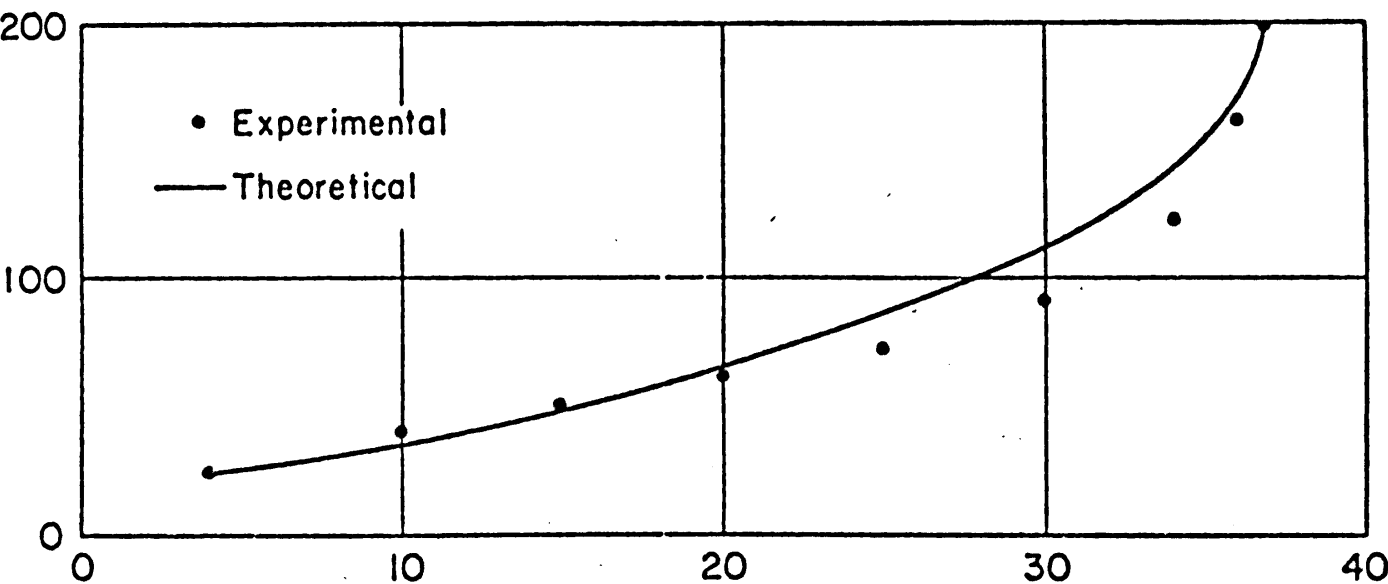

Number of Cycles, $\mathrm{N}$

Figure 4. Pore Water Pressure versus Number of Cycles

\section{CONCLUSIONS}

Within the scope and limitations outlined in the foregoing study, the following conclusions can be advanced:

1. Endochronic theory provides a realistic mathematical model for the densification and hysteretic behavior of sand subjected to cyclic deviatoric strains. The theory may also be applied for arbitrary strain histories.

2. The basic extension with regard to previous applications of endochronic theory to metals is the presence of densification (negative dilatancy) and hydrostatic pressure sensitivity; these two features are shared with the previous formulation of endochronic theory for concrete.

3. The intrinsic time parameter may be associated with the rearrangement measure, which physically represents accumulated rearrangements of particle configurations.

4. The combination of the present theory with the previous formulation for inelastic densification in a two-phase medium yields a realistic model for liquefaction of an undrained saturated sand. 
1. Bazant, Z. P. (1974a), "A New Approach to Inelasticity and Fallure of Concrete, Sand, and Rock: Endochronic Theory", Proceedings of the Eleventh Annual Meeting, Society of Engineering Science, G. J. Dvorak, Editor, Duke University, Durham, North Carolina, pp 158-159.

2. Bažant, Z. P. (1974b), "Theory of Creep and Shrinkage in Concrete Structures: A Précis of Recent Developments", Mechanics Today, Volume 2, Pergamon Press, pp 1-93.

3. Bazant, Z. P., and Bhat, P.(1974), Endochronic Theory of Inelasticity and Failure of Concrete, Structural Engineering, Report No. NSF-GK26030-74-2, Dept. of Civil Engineering, Northwestern University, Evanston, Illinois, November 1974.

4. Bažant, Z. P., and Krizek, R. J. (1975), "Saturated Sand As an Inelastic Two-Phase Medium", Journal of the Engineering Mechanics Division, American Society of Civil Engineers (in press).

5. Biot, M. A. (1956), "Theory of Propagation of Elastic Waves in FluidSaturated Porous Solid-I. Low-Frequency Range," Journal of the Acoustical Society of America, Volume 28, 168-178.

6. Biot, M. A. (1957), "The Elastic Coefficients of the Theory of Consolidation", Journal of Applied Mechanics, Volume 24, 594-601.

7. Coon, M. D, Evans, R. J. (1971), "Recoverable Deformation of Cohesionless Soils", Journal of the Soil Mechanics and Foundations Division, American Society of Civil Engineers, Volume 97, Number SM2, pp. 375-391.

8. Finn, W. D., Liam, Bransby, P. L., and Pickering, D. J. (1970), "Effect of. Strain History on Liquefaction of Sand", Journal of the Soil Mechanics. and Foundations Division, American Society of Civil Engineers, Volume 96, Number SM6, pp 1917-1934.

9. Lade, P. V., and Duncan, J. M. (1975), Plastic Stress-Strain Theory for Cohesionless Soil, Personal Communication.

10. Martin, G. R., Finn, W. D. L., and Seed, H. B. (1974), Fundamentals of Liquefaction Under Cyclic Loading, Soil Mechanics Series No. 23, Department of Civil Engineering, University of British Columbia, Vancouver, Canada.

11. Richart, F. E., Hall, J. R., and Woods, R. D. (1970), Vibrations of Soils and Foundations, Prentice-Hall, Inc., Englewood Cliffs, New Jersey.

12. Silver, M. L. (1S74), Unpublished Data, Personal Communication.

13. Silver, M. L. and Seed, H. B. (1971), "Volume Changes in Sands During Cyclic Loadings", Journal of the Soll Mechanics and Foundations Division, American Society of Civil Engineers, Volume 97, Number SM9, pp. $1171-1182$.

14. Valanis, K. C. (1971), "A Theory of Viscoplasticity without a Yield Surface", Archivum Mechaniki Stosowanej, Volume 23, Number 4, pp 517-533. 